\title{
High Likelihood of Meningitis with Late Onset Septicemia in Newborn
}

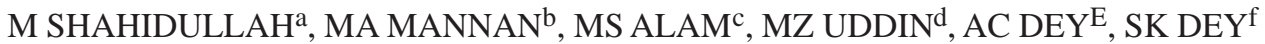

\begin{abstract}
Summary:
Neonatal meningitis must be recognized and treated quickly to prevent death or disability. Incidence of neonatal meningitis with late onset sepsis is higher in developing countries than those of resource-rich countries. In neonates signs and symptoms of serious infections are often obscured and clinical examination cannot distinguish among septic babies with or without meningitis. Clinicians often differ whether neonates undergo lumber puncture or not to distinguish septic babies with or without meningitis. Abnormal CSF findings are often used to detect neonatal meningitis and determine the type and length of antibiotic therapy with proven sepsis and meningitis cases. This study was conducted to evaluate the bacterial meningitis among the late onset sepsis in newborns and to identify the clinical manifestations that can distinguish septicemia from meningitis in neonates.
\end{abstract}

Total 1706 admitted patient in NICU of Bangabandhu Sheikh Mujib Medical University from January 2007 to

\section{Introduction:}

Bacterial meningitis continues to be a serious problem with high morbidity and mortality. ${ }^{1-3}$ Neonatal bacterial

a. Prof. Mohammod Shahidullah, Chairman, Department of Neonatology \&, Pro-VC, BSMMU.

b. Dr. M.A. Mannan, Associate Professor, Department of Neonatology, BSMMU.

c. Dr. Mohammad Shakhawat Alam, FCPS Student, Department of Neonatology, BSMMU, Dhaka.

d. Dr. Mohammad Zahir Uddin, FCPS Neonatology Student, Department of Neonatology, BSMMU, Dhaka. .

e. Dr. Arjun Chandra Dey, Assistant Professor, Department of Neonatology, BSMMU.

f. Dr. Sanjoy Kumer Dey, Assistant Professor, Department of Neonatology, BSMMU.

Address of Correspondence: Dr. M.A. Mannan, Associate Professor, Department of Neonatology, Bangabandhu Sheikh Mujib Medical University, Dhaka, Bangladesh, Phone: 01715-055506,, Email: neonatal@bdcom.com,

Received : 14 August, 2010

Accepted: 20 December, 2011
December 2009 were evaluated retrospectively. Among the 133 (27.94\%) cases of suspected late onset sepsis 47(35.33\%) were proven sepsis, $63(47.37 \%)$ were probable sepsis and 23(17.29\%) cases were clinical sepsis based on clinical features laboratory reports and blood cultures. Among the proven sepsis 12(42.85\%) cases were found to have definitive bacterial meningitis and 16(57.15\%) were probable bacterial meningitis. Among the provable sepsis only 1(12.50\%) cases were found to have definite bacterial meningitis and 7 (87.50\%) cases were probable bacterial meningitis. There were no meningitis have found among the clinical sepsis.

Neonatal meningitis frequently occurred in late onset sepsis. The most frequent presenting clinical features for meningitis cases are more or less similar to those of septicemic cases.

The data of the study suggest that newborns with a positive blood culture are significantly more likely to have meningitis than those with a negative blood culture.

(J Bangladesh Coll Phys Surg 2012; 30: 17-23)

meningitis occurs relatively frequently in newborn infant with incidence of 0.2 to 0.5 per 1000 live births based on geographically defined population. ${ }^{4}$ Incidence of neonatal meningitis with late onset sepsis is higher in developing countries of South Asian sub-continent than resource- rich countries. ${ }^{5}$

Neonates receiving prolonged intensive care who are prone to nosocomial infection are in high risk group of developing meningitis. ${ }^{6}$ Previous studies have reported rates of hospital acquired neonatal infections that are 3 to 20 times higher in resource-poor countries than those of resource-rich countries. ${ }^{7}$ There were $49 \%$ cases of all sepsis cases occurred between the age 8 to 28 days. ${ }^{8}$ About $20 \%$ - $30 \%$ of neonatal septicemia whether early or late, is complicated by bacterial meningitis. ${ }^{9-10}$ Neonates with Early Onset sepsis with obstetric risk factors or respiratory distress alone, lumber puncture may be delayed but lumber puncture should be performed in babies with late onset sepsis because organisms in CSF often affects treatment of choice and duration of the therapy. ${ }^{11}$ 
Neonatal meningitis must be recognized and treated quickly to combat preventable death or disability. In Neonates signs and symptoms of serious infections are often obscured and clinical examination cannot distinguish septic babies with or without meningitis. The causative agents of neonatal sepsis vary between geographical areas and with time in any particular locality. ${ }^{12}$ In North America and Europe, the prevalent bacterial agents of early onset sepsis are Group B Streptococcus (GBS), Listeria Monocytogenes and E. Coli and those of late onset sepsis include Coagulase Negative Staphylococcus (CONS), Kebsiella, E. Coli. ${ }^{13-}$ ${ }^{14}$ In India Shashikala S, Tallur et al. Showed Klebsiella was the commonest organism causing neonatal meningitis. ${ }^{15}$ On the contrary, blood culture may also be negative in meningitis, as reported in 13-15 percent of patients with CSF culture-proven definite bacterial meningitis among the provable sepsis. ${ }^{16} \mathrm{CSF}$ studies are recommended for all infants who are found to have positive blood cultures. ${ }^{17}$ The importance of Lumber Puncture (LP) as a part of the diagnostic evaluation of the neonate with suspected sepsis has been the subject of debate and clinical practice varies. ${ }^{11,16,18}$

Neonatologists or Pediatricians often ignore the LP when evaluating the newborn infants. Reasons stated include the perceived low risk of meningitis versus the risk of the procedure in the often unstable patient. ${ }^{11,18-}$ ${ }^{19}$ We suspected that there would be differences among the centers in the frequency with which an LP was performed and speculated that meningitis may be underdiagnosed because of the failure to perform routinely LP in neonates with suspected late onset sepsis. The aim of this study was to establish the importance of concomitant central nervous system infection in cases of late onset neonatal sepsis. Specifically, we sought to: (i) evaluate the bacterial meningitis among the late onset sepsis in newborns, (ii) identify the clinical manifestations that can distinguish late onset septicemia alone from those complicated by meningitis in neonates.

\section{Materials and Methods:}

Medical records of all neonates admitted in the Department of Neonatology, Bangabandhu Sheikh Mujib Medical University (BSMMU) from January 2007 to December - 2009 were evaluated. Clinical data of the infants and laboratory findings were examined retrospectively.
Late Onset Sepsis (LOS) was defined as infection occurring after 7 days of age up to 28 days of age with two or more clinical features of sepsis like reluctant to feed, lethargy, temperature instability, axillary temperature $<36 \mathrm{C}$ or $>38 \mathrm{C}$, feeding intolerance, apnoea, respiratory distress, capillary filling time $>3$ seconds on the forehead or sternam, vomiting, diarrhea, abdominal distension, rapid rise of serum bilirubin $>15$ $\mathrm{mg} \%$ in the absence of blood group incompatibility, petechiae or bleeding diathesis, mottling, bulged fortanelle and convulsion etc were present.

Laboratory markers of CBC and CRP level were considered abnormal if- total Leukocyte count $<5000 /$ cmm or $>25000 / \mathrm{cmm}$, Absolute neutrophil count $<1500 /$ cmm, Immature total neutrophil ratio $>0.2$ and $\mathrm{PBF}$ showed nucleated RBC with presence of toxic granules and band and thrombocytopenia (platelet count $<1500000 / \mathrm{cmm}$

CRP had measured quantitatively. A positive test result indicates a CRP level more than $6 \mathrm{mg} / \mathrm{L}$.

A neonate with LOS was diagnosed as a case of meningitis if any significant bacteria was isolated from CSF and/or the fluid contained more than 30 leukocytes/ $\mathrm{mm}^{3}$, protein e" $200 \mathrm{mg} / \mathrm{dl}$ and d"40 mg/dl of sugar.

Positive blood and CSF cultures were considered the 'gold standard' against the laboratory values of CBC and CRP level.

\section{Working definition of LOS to be included:}

1) Blood culture usually yields growth of organism though in many cases it may be negative along with presence of clinical features and positive other laboratory values of CBC and CRP level and termed as proven sepsis.

2) If culture results were negative but other laboratory tests were positive along with two or more clinical features of sepsis were present and termed as probable sepsis.

3) If culture results and other laboratory tests were negative but two or more clinical features of sepsis were present and termed as clinical sepsis.

Working definition of bacterial meningitis with LOS to be included:

1) Definite bacterial meningitis was diagnosed if there were growth of organisms from CSF culture along 
with other laboratory values of cytological, Gram staining and biochemical level.

2) Probable bacterial meningitis was diagnosed if no pathogens were obtained from CSF culture but other laboratory values of cytological, Gram staining and biochemical level were present.

\section{Criteria to be excluded:}

Neonates with gross congenital anomalies and those having birth weight less than 800 gms were excluded from the study.

All patients received standard empirical treatment for neonatal sepsis according to protocol of proven sepsis 14 days, probable sepsis for 7-10 days and clinical sepsis for 7days.All neonates with proven or probable meningitis received treatment for 21 days. Subsequently antibiotics were changed depending on the culture sensitivity report and/or the clinical condition.

\section{Data collection and analysis:}

Data were collected from medical records admitted in NICU from January 2007 to December 2009. All the data were analyzed with SPSS software. Clinical features of LOS were compared with the features of LOS alone and those complicated by bacterial meningitis. P values were reached from Chi square test with $95 \%$ CI.

\section{Results:}

A total of 1706 neonates were admitted in the BSMMU NICU from January 2007 to December 2009.Among them 476 (27.90\%) neonates were cases of suspected sepsis (both early and late onset sepsis)

Out of 476 cases 133 (27.94\%) were found to have LOS of which 47 (35.34\%) cases were Proven sepsis, 63 (47.37\%) cases were Probable sepsis and 22 (16.54\%) cases were Clinical sepsis.

There were 4 cases from the proven sepsis and 12 cases from the probable sepsis were excluded from the study as lumbar puncture could not be done due to life threatening condition and/or congenital anomalies

The baseline characteristics of the babies has shown in Table -I

Male: Female ratio was 1.2:1. The mean gestational age (GA) was $34 \mathrm{wk} \pm .2$ (range-28-40wks) weeks and mean birth weight was 1700 gms \pm 50 (range-9004080gms). Among 117 cases of Late Onset Sepsis 65(55.56\%) cases were inborn and 68(58.11\%) cases were delivered by LUCS.

Clinical features of the cases were analyzed and shown in the Table-II.

The common features were found to have- reluctant to feed, lethargy, feeding intolerance temperature instability, apnoeas, jaundice, respiratory distress etc.

Table-I

The baseline characteristics of the babies $(n=117)$

\begin{tabular}{cll}
\hline \multicolumn{1}{c}{ Sex } & Male- & $64(54.70 \%)$ \\
& Female - & $53(45.29 \%)$ \\
Birth weight & $<1500$ gms- & $47(40.17 \%)$ \\
& $1500-<2500$ gms- & $38(32.48 \%)$ \\
Gestational age (GA) & $2500 \mathrm{gm}->2500 \mathrm{gms}$ & $32(27.35 \%)$ \\
& $<30$ wks - & $37(31.62 \%)$ \\
Mode of delivery & $30-34$ wks - & $35(29.91 \%)$ \\
Place of delivery & $35-<37$ wks - & $24(20.51 \%)$ \\
& $37->37$ wks- & $21(17.96 \%)$ \\
& LUCS - & $68(58.11 \%)$ \\
& NVD - & $49(41.89 \%)$ \\
& Inborn- & $65(55.56 \%)$ \\
& Out born - & $52(44.44 \%)$ \\
\hline
\end{tabular}


Table - II

Clinical features of both Septic and Meningitic cases

\begin{tabular}{lccc} 
& \multicolumn{2}{c}{ Total 117 } & \\
\cline { 2 - 3 } & $\begin{array}{c}\text { Septic Baby } \\
(\mathrm{n}=81)\end{array}$ & $\begin{array}{c}\text { Meningitic Baby } \\
(\mathrm{n}=36)\end{array}$ & P-Value \\
& Number (Percentage) & Number (Percentage) & \\
\hline Reluctant to feed & $48(59.25 \%)$ & $17(47.22 \%)$ & $0.226 \mathrm{NS}$ \\
Lethargy & $45(55.56 \%)$ & $18(50.00 \%)$ & $0.577 \mathrm{NS}$ \\
Feeding intolerance & $46(56.79 \%)$ & $21(58.33 \%)$ & $0.876 \mathrm{NS}$ \\
Temperature Instability & $29(35.80 \%)$ & $19(52.78 \%)$ & $0.084 \mathrm{NS}$ \\
Apnea & $31(38.27 \%)$ & $14(38.89 \%)$ & 0.949 NS \\
Abdominal distention & $40(49.38 \%)$ & $21(58.33 \%)$ & $0.371 \mathrm{NS}$ \\
Vomiting & $39(48.14 \%)$ & $22(61.11 \%)$ & $0.195 \mathrm{NS}$ \\
Jaundice & $17(20.98 \%)$ & $8(22.22 \%)$ & $0.880 \mathrm{NS}$ \\
Convulsion & $6(07.40 \%)$ & $5(13.89 \%)$ & $0.267 \mathrm{NS}$ \\
Respiratory distress & $23(28.39 \%)$ & $12(33.33 \%)$ & $0.590 \mathrm{NS}$ \\
Bulged fontanel & $3(03.70 \%)$ & $3(08.33 \%)$ & $0.294 \mathrm{NS}$ \\
High pitch cry & $0(0 \%)$ & $1(02.77 \%)$ & $0.131 \mathrm{NS}$ \\
\hline
\end{tabular}

P value reached from Chi square test with 95\% CI , NS = Not Significant

Features did not differ in the cases with or without meningitis. P values revealed no significant differences in clinical features among the septicemia cases alone or complicated by meningitis.

The most frequent presenting clinical features for meningitis cases were more or less similar to those of septicemic cases; reluctant to feed (59\% vs $47 \%$ ), lethargy ( $56 \%$ vs $50 \%$ ), feeding intolerance ( $57 \%$ vs $58 \%$ ), apnoea (38\% vs $39 \%$ ), abdominal distention (49\%vs 58\%), vomiting (48\% vs 61\%) and temperature instability (36\% vs 53\%).

There were 3 (8.33\%) cases found to have bulged fontanels and 5 (13.89\%) cases convulsion among meningitic patients. Out of the entire septic baby 6 (7.40\%) cases were found to have convulsion and 3 (3.70\%) cases were bulged fontanelles.

Among the 43 cases of Proven sepsis, 28 (65.11\%) cases were found to have meningitis of which 12 (42.86\%) cases were Definite bacterial meningitis and 16 (57.14\%) cases were Probable bacterial meningitis based on either by CSF culture positive and/or CSF abnormality.

Among the 51 cases of Probable sepsis, 8 (15.68\%) cases were found to have meningitis of which $1(12.5 \%)$ case had Definite bacterial meningitis as CSF culture was positive. Other 7 (87.5\%) cases had Probable bacterial meningitis as abnormal CSF laboratory report but CSF culture were negative.

Among the 22 cases of Clinical sepsis with strongly clinical suspicion of meningitis, there were no cases found to have Definite and/or Probable bacterial meningitis.

Finally out of 117 cases of LOS, Bacterial meningitis were found to have in $36(30.76 \%)$ cases, shown in Figure I.

Among all the cases of culture positive LOS 18(41.86) cases Klebseilla, 17(39.53\%) cases E.coli, 3(6.98\%) cases Pseudomonus, 3(6.98\%) cases Acinatobacter and 2(4.65\%) cases Enterobacter were found.

Out of all the 13 (36.11\%) cases of definite bacterial meningitis yielded growth of bacteria of which there were $7(53.85 \%)$ cases of E.Coli, 4 (30.77\%) cases of Klebseilla and 2 (15.38\%) cases of Pseudomonas which had matched with identification and antibiotic susceptibility profile as their corresponding blood culture ,shown in the Table-III.

Among the 36 cases of bacterial meningitis 12(33.33\%) cases were found to have concomitant blood and CSF 


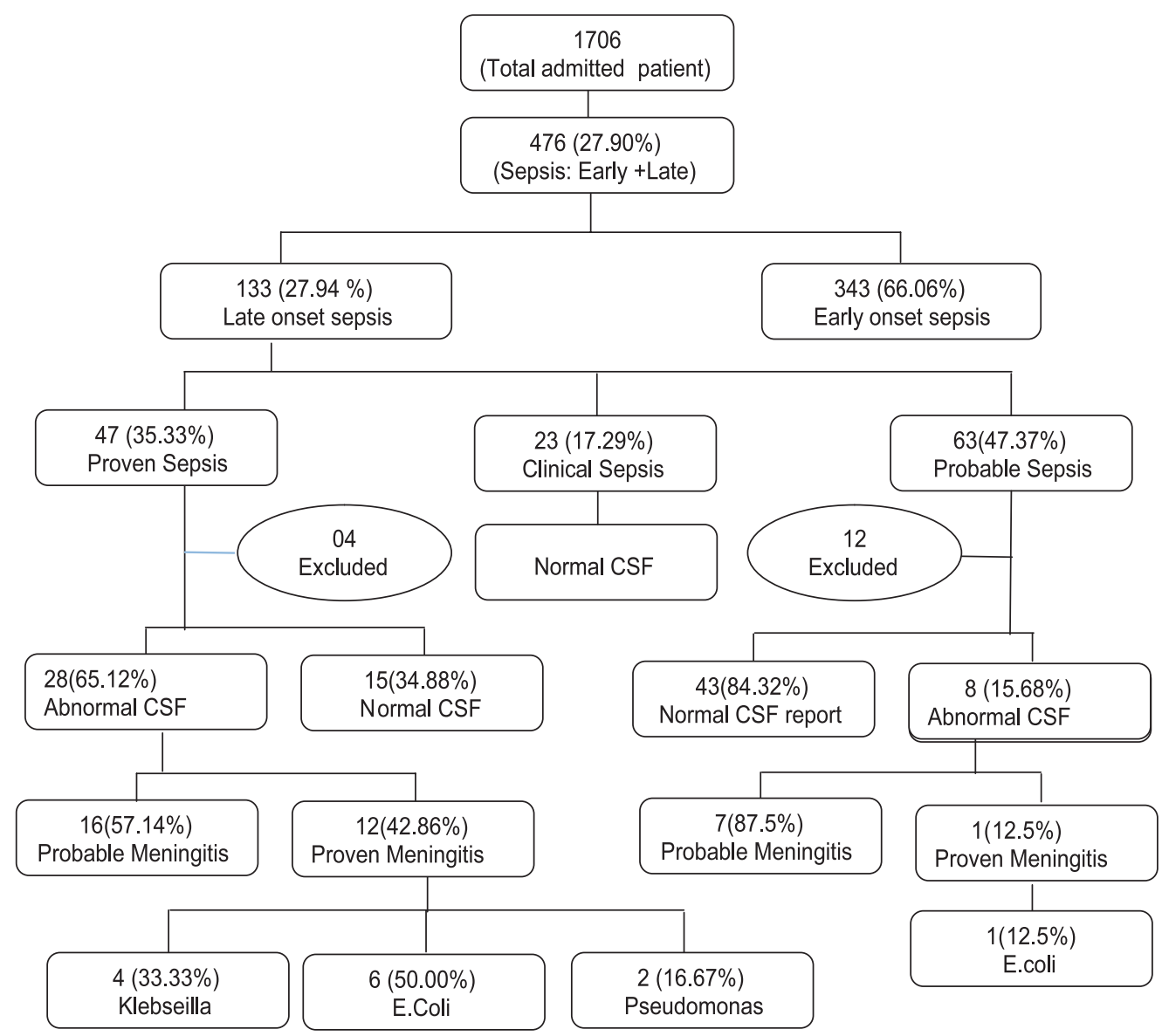

Fig-I: Flow chart of the Results

Table-III

Distribution of pathogen in the blood and CSF

Name of Organism

Klebseilla

E.coli

Pseudomonas

Acinetobacter

Enterobacter
Septic Baby ( $\mathrm{n}=43)$

Number (Percentage)
Meningitic Baby ( $\mathrm{n}=13)$

Number (Percentage)

$18(41.86 \%)$
$17(39.53 \%)$
$3(6.98 \%)$
$3(6.98 \%)$
$2(4.65 \%)$

culture positive and $1(2.79 \%)$ cases where CSF culture was positive but blood culture was negative.

In neonates with both positive blood and CSF cultures, there was no discordance among the organism isolated.

\section{Discussion:}

Neonatal meningitis has a high mortality and significant risk of morbidity and neurological sequelae. But the diagnosis is often difficult or sometimes delayed as clinical features are non specific and LP is not performed routinely.

In some studies it has been shown that $20-30 \%$ cases of neonatal sepsis whether early or late were complicated by neonatal meningitis. In this study we have found $30.76 \%$ cases of neonatal meningitis among the suspected cases of LOS. ${ }^{9} 1020$ 
The incidence was also very high in the preterm and low birth weight babies which was inversely related to gestational age and birth weight consistent with previous studies. $^{13,21}$

Commonest organism of neonatal meningitis in the developed countries is Group B Streptococcus (GBS) which is not quite similar in Bangladesh. Studies reported E. coli, Klebseilla to be the major causative organism of neonatal meningitis. ${ }^{20-21}$ This study has been correlated the previous studies of the country in regard to neonatal sepsis where the organisms were found to have E-coli, Klebseilla, and Pseudomonas as the important organisms of neonatal meningitis as comparable to other studies of Bangladesh. ${ }^{20}$

Since most cases bacterial pathogens involved in the neonatal sepsis are commonly the same for neonatal meningitis in a given environment. This observation has been corroborated by parallel studies which also identified same organisms as the most frequent pathogens of neonatal sepsis in the same unit. In this study all the culture positive neonatal meningitis cases had the same organisms as in the blood indicating that these were the complication of LOS as in other studies. ${ }^{13,20}$

In this study there were $12(33.33 \%)$ cases where simultaneously blood culture and CSF culture were positive but in other studies there were 50 - 66\% cases had concomitant blood culture and CSF culture were positive. ${ }^{20,22}$

As reported by others 13 -38\% cases, where blood culture was negative but organisms were isolated in the CSF as culture proven meningitis. ${ }^{13} 22$ This study also showed $2.79 \%$ cases among the total bacterial meningitis where organisms were not isolated from the blood but isolated from CSF culture.

In neonates with both positive blood and CSF cultures, the organisms isolated were discordant in 3.5\% of cases but in this study there were no discordance of isolated organism as found in other study. ${ }^{20,22}$

Neonates with a positive Blood culture were significantly and more likely to have meningitis than those with a negative blood culture and lumbar puncture should be considered as a part of routine investigation in late onset sepsis.$^{23-25}$
The necessity of performing lumbar puncture in newborn has been debated because of the perceived risks of procedure in very sick infant but other studies have shown that the incidence of meningitis secondary to lumbar puncture is very low and there are no increased risks of death that had a lumbar puncture. ${ }^{13,25-26}$ In this study there were no data of meningitis or death secondary to lumbar puncture during the study period.

Limitation of this study was that the LP was done sometime after receiving antibiotics.

Nonetheless the data confirmed that it is very important to an LP along with blood culture for all suspected cases of LOS.

\section{Conclusion:}

Neonatal meningitis is not uncommon in late onset sepsis. The presenting clinical features for meningitis cases are more or less similar to those of septicemic cases. The data of the study suggest that neonates with positive blood culture LOS are more likely to have meningitis than those with negative blood culture.

\section{References:}

1. Davies PA, Rudd PT. Neonatal meningitis. London, United Kingdom: MacKeith press; 1994:1-77.

2. Holt DE, Halket S, de Louvois J, Harvey D. Neonatal meningitis in England and Wales: 10 years on. Arch Dis Child Fetal and Neonatal Ed.2001; 84:85-89.

3. Harvey D, Holt DE. Bacterial meningitis in the newborn: a prospective study of mortality and morbidity. Semin Perinatol.1999;23:218-25

4. Overall JC. Neonatal bacterial meningitis. J.Pediatr.1970;76:499-511

5. Tiskumara R, Fakharee SH, Liu CQ, Isaacs D. Neonatal infections in Asia. Arch Dis Child Fetal Neonatal Ed.2009; 94: F144-48.

6. Anne G. Bacterial sepsis and Meningitis. Seminars in Fetal \& Neonatal Medicine.1996; 1: 147-59.

7. Zaidi A, Huskins C, Thaver D. Hospital-acquired infection in developing countries.Lancet.2005; 365: 1175-89.

8. Greenberg D, Shinwell ES, Yagupsky P. A prospective study of neonatal sepsis and meningitis in Southern Israel. Pedatr. Infect. Dis. J.1997;16 (8);768-73

9. Isaacs D, Moxon ER. Neonatal infections. Oxford: Butterworth-Heineman. 1991:57.

10. De Louvois J, Harvey D. Infection in the newborn. London: John Wiley and sons, 1990:107-15.

11. McIntyre P, Ishacs D. Lumbar puncture in suspected neonatal sepsis. J. Pediatri Child Health .1995; 31: 1-2. 
12. Dawodu A, al Umran K, Twum-Danso K. A case control study of neonatal sepsis: Experience from Saudi Arabia. J. Trop. Pediatri. 1997; 43(2)84-88.

13. Hristeva L, Booy R, Bowler I, Wilkinson AR. Prospective surveillance of neonatal meningitis. Arch Dis Child .1993;69:14-18

14. Synnott MB, Morse Dale L, Susan M Hall. Neonatal meningitis in England and Wales. A review of routine national data .Arch. Dis. Child. Fetal Neonatal Ed. 1994; 71(2):7580

15. Shashikala ST, Kasturi AV, Shobha D. Nadgir. Clinicobacteriological study of neonatal meningitis. Indian J. of Pediatrics .2000; 67:169-74.

16. Visser VE, Hall RT. Lumbar punctures in the evaluation of suspected neonatal sepsis. J. Paediatr.1980; 66:1063-67.

17. Guerina NG. Bacterial and fungal infections. In: Cloherty JP, Stark AR (eds). Manual of Neonatal Care (4 ${ }^{\text {th }}$ edition) LippincottRaven, Philadelphia 1997:271-85.

18. Schwersenski J, McIntyre L, Bauer CR. Lumbar puncture frequency and CSF analysis in the neonates. Am. J. Dis Child .1991;145:54-58
19. MacMahon P, Jewes L, de Louvois J. Routine lumbar puncture in the newborns - are they Justified? Eur. J. Pediatrics .1990; 149:797-799.

20. Mahbubul H, Nawshad UA. Azad Chawdhury MAK, Gary L D, Samir K. Septicaemic neonates Without Lumber Puncture; What are we missing? J. Trop. Pediatrics 2005 June

21. Laving AM., Musoke RN, Revathi G. Neonatal bacterial meningitis at the newborn unit of Kenyatta national hospital. East Afr Med J.2003;80:458-62

22. Kenneth TF. Lenfestey R, Jennifer S. Neonatal Meningitis: What Is the Correlation Among Cerebrospinal Fluid Culture, Blood Cultures, and Cerebrospinal Fluid Parameters? Pediatrics 2006; 117; 1094-00.

23. Francis BM, Gilbert GL. Survey of neonatal meningitis in Australia: 1987-1989. Med. J. Aust.1992; 156; 240-43

24. . Barbana J. Stoll, Hansen N, Avroy A, Kenneth W. To Tap or Not To Tap: High Likelihood of meningitis without sepsis among VLBW infants. Pediatrics 2004; 113; 1181-1186

25. Malbon K, Mohan R, Nicholl R. Should a neonate with possible late onset infection always have a lumbar puncture? Arch. Dis. Child .2006; 91:75-76

26. Mobeen H. Rathore. A VLBW infant with suspected sepsis: To Tap or Not to Tap-That is the question. AAP. Grand rounds 2004; 12: 9-9. 